\title{
Cross-Bridge Kelvin Resistor Structures for Reliable Measurement of Low Contact Resistances and Contact Interface Characterization
}

\author{
Natalie Stavitski, Student Member, IEEE, Johan H. Klootwijk, Senior Member, IEEE, Henk W. van Zeijl, \\ Alexey Y. Kovalgin, and Rob A. M. Wolters
}

\begin{abstract}
The parasitic factors that strongly influence the measurement accuracy of Cross-Bridge Kelvin Resistor (CBKR) structures for low specific contact resistances $\left(\rho_{c}\right)$ have been extensively discussed during last few decades and the minimum of the $\rho_{\mathrm{c}}$ value, which could be accurately extracted, was estimated. We fabricated a set of various metal-to-metal CBKR structures with different geometries, i.e., shapes and dimensions, to confirm this limit experimentally and to create a method for contact metal-tometal interface characterization. As a result, a model was developed to account for the actual current flow and a method for reliable $\rho_{\mathrm{c}}$ extraction was created. This method allowed to characterize metal-to-metal contact interface. It was found that in the case of ideal metal-to-metal contacts, the measured CBKR contact resistance was determined by the dimensions of the two-metal stack in the area of contact and sheet resistances of the metals used.
\end{abstract}

Index Terms-Cross-bridge Kelvin resistor (CBKR), metal-tometal contacts, specific contact resistance.

\section{INTRODUCTION}

$\mathbf{C}$ ROSS-BRIDGE Kelvin Resistor (CBKR) structures are the most widely used test structures to characterize metal-semiconductor contacts in the planar devices of VLSI technology [1], [2]. On the other hand, CBKR was found to be very sensitive to lateral current crowding around the contact when the contact window is smaller than the underlying layer. Several simulations and correction methods were introduced in order to account for this current crowding effect [3]-[6]. However in the low resistance range, the extracted silicide-to-silicon specific contact resistance $\left(\rho_{c}\right)$ values, obtained using CBKR structures, were still orders of magnitude different from the results obtained using other methods [2]. An explanation of this phenomenon is the accuracy problems during the data extraction using CBKR structures in the range of $\sim 10^{-8} \Omega \cdot \mathrm{cm}^{2}$ and below [7]. In this case, the lateral current flow around

Manuscript received June 30, 2008; revised October 21, 2008. Current version published February 04, 2009.

N. Stavitski and A. Y. Kovalgin are with the MESA+ Institute for Nanotechnology, Chair of Semiconductor Components, University of Twente, Postbox 217,7500AE Enschede, The Netherlands (e-mail: n.stavitski@utwente.nl).

J. H. Klootwijk is with the Philips Research, High Tech Campus 4, $5656 \mathrm{AE}$ Eindhoven, The Netherlands.

H. W. van Zeijl is with the DIMES, Delft University of Technology, Feldmannweg 17, 2628 CT Delft, The Netherlands.

R. A. M. Wolters is with the MESA+ Institute for Nanotechnology, Chair of Semiconductor Components, University of Twente, Postbox 217, 7500AE Enschede, The Netherlands and also with NXP Research Eindhoven, High Tech Campus 4, 5656 AE Eindhoven, The Netherlands.

Digital Object Identifier 10.1109/TSM.2008.2010746 the contacts gives rise to an even higher additional resistance [8], [9]. This effect becomes more pronounced for a lower $\rho_{\mathrm{c}}$ and a higher sheet resistance $\left(R_{\mathrm{sh}}\right)$ of the underlying layer. Simulations show that for $\rho_{\mathrm{c}}<10^{-7} \Omega \cdot \mathrm{cm}^{2}$ the extracted $\rho_{\mathrm{c}}$ can differ by one or two orders of magnitude from the actual value [6]. Moreover, the trend in the modern technology of high-density integrated circuits is toward lower $\rho_{\mathrm{c}}$ and higher $R_{\mathrm{sh}}$ values, due to the shallower junctions. This will further complicate the interpretation of CBKR measurement results.

Our research is therefore concerned with finding the minimum contact resistance, which can be obtained experimentally using CBKR test structures, and developing a correction model to account for the actual current flow. In addition this method should allow to characterize metal-to-metal contact interfaces, crucial for contact manufacturing. For that purpose, CBKR structures of different geometries, i.e., dimensions and shapes of the contact area were designed and manufactured. These structures were evaluated for metal-to-metal contacts without an interface, to assure the case of very low contact resistances, and for metal-to-metal contacts with an interface present, to demonstrate the ability of the interface characterization.

\section{Measurement Technique and Test Structures DESCRIPTION}

A standard four-terminal CBKR test structure is used to determine $\rho_{\mathrm{c}}$ of metal-to-metal contacts (Fig. 1). The measurement principle consists of forcing the current $(I)$ between pads 1 and 2 and measuring the voltage drop $\left(V_{34}\right)$ between pads 3 and 4 . The actually measured Kelvin resistance $R_{\mathrm{k}}$ can then be found as

$$
R_{\mathrm{k}}=\frac{V_{34}}{I} .
$$

In the 1-D Model approach [4], the specific contact resistance can be calculated directly from the contact area $A$ and $R_{\mathrm{k}}$, assuming that the resistance due to the voltage drop across the actual contact $\left(R_{\mathrm{c}}\right)$ equals $R_{\mathrm{k}}$ :

$$
\rho_{\mathrm{c}}=R_{\mathrm{c}} A=R_{\mathrm{k}} A .
$$

The 1-D Model does not account for the current flowing in the overlap region $(\delta)$ of the underlying layer (Fig. 1), when $\delta>$ 0 . In that case the so-called 2-D Model should be applied [4]. The analytical model by Schreyer and Saraswat was used in this 


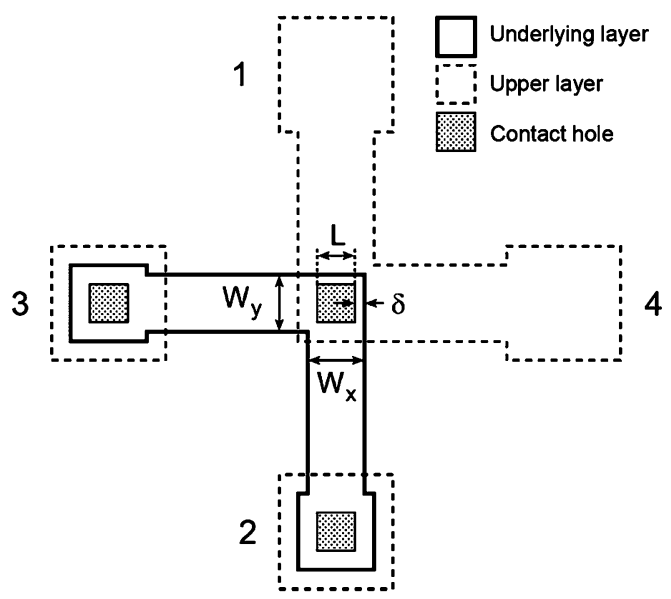

Fig. 1. Four-terminal CBKR structure with geometry parameters definition. In our structures, the contact geometry parameters $(\delta$ and $L)$ for both layers are identical, unless mentioned otherwise.

study as a starting point for the correction. The measured $R_{\mathrm{k}}$ is then a sum of the $R_{\mathrm{c}}$ and the resistance due to the current flow around the contact in the overlap region $\left(R_{\text {geom }}\right)$ (3). The $\rho_{\mathrm{c}}$ can further be extracted from (4), where $R_{\mathrm{sh}}$ is the sheet resistance of the underlying layer. The contact geometry parameters are defined in Fig. 1:

$$
\begin{aligned}
& R_{\mathrm{k}}=R_{\mathrm{c}}+R_{\text {geom }} \\
& R_{\mathrm{k}}=\frac{\rho_{\mathrm{c}}}{A}+\frac{4 R_{\mathrm{sh}} \delta^{2}}{3 W_{\mathrm{x}} W_{\mathrm{y}}}\left[1+\frac{\delta}{2\left(W_{\mathrm{x}}-\delta\right)}\right] .
\end{aligned}
$$

In order to verify the validity of the results obtained, the CBKR structures were designed to cover a wide range of contact sizes (i.e., length $L$ for square contacts and diameter $D$ for round contacts) and $\delta$. Some of the structures were designed with two $\delta$, different for the lower and upper metal layers: $\delta_{L}$ and $\delta_{U}$, respectively. To exclude the uncertainty in the definition of $\delta$ in the case of round contacts, the metal tap width ( $V_{\text {tap }}$, Fig. 2(b)) was varied as well. The details are summarized in Table I.

The sheet resistances of both the lower and upper metal layers were measured using Van-der-Pauw (VDP) structures, located on the same chip. The chip layout and an example of the square and round CBKR structure are presented in Fig. 2.

Five dies per wafer were measured (CBKR and VDP). The obtained results were that close, that the measurement error bounds were invisible in all graphs.

\section{TeSt Structures Fabrication}

The (100) p-type Si wafers with a 1- $\mu$ m-thick thermal oxide were used to fabricate the test structures for this study. First, a 0.675 or $1.4-\mu \mathrm{m}$-thick Al layer was sputtered and patterned using I-line lithography and plasma etching. Then, a $0.8-\mu \mathrm{m}$-thick layer of $\mathrm{SiO}_{2}$ was deposited by PECVD and the contact holes were opened. Prior to the second $\mathrm{Al}$ deposition, the contacts were either in situ RF-precleaned to create noninterface metal-to-metal contacts or this preclean procedure was skipped to obtain the contacts with interface present. The second $\mathrm{Al}$ layer of 0.675 or $1.41 \mu \mathrm{m}$ was sputtered and patterned as the upper metallization layer, including the bond pads. (a)

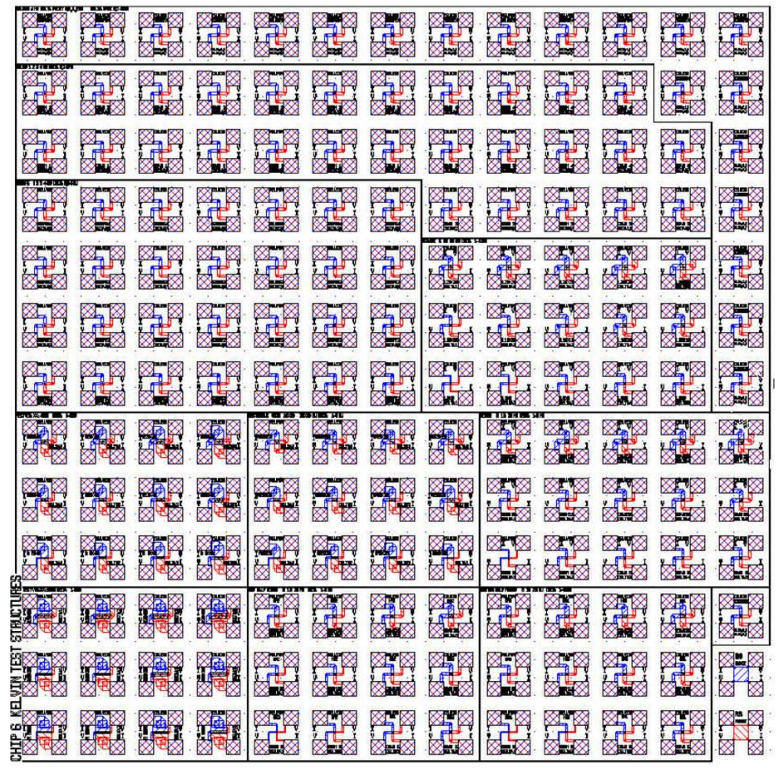

(b)

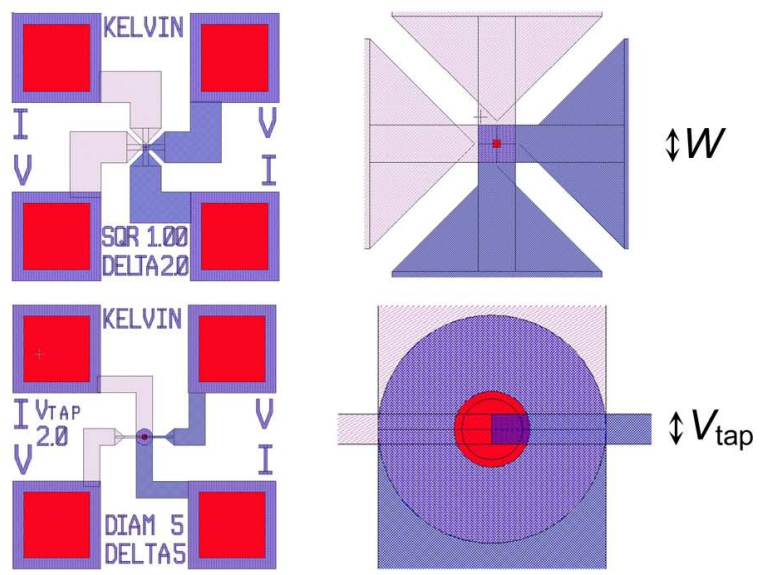

Fig. 2. (a) The chip layout, including CBKR and VDP structures. (b) An example of the newly-designed square and round CBKR structures. The complete structure including the bond pads is on the left- and a blow up of the actual contact is on the right-hand side.

TABLE I

IMPORTANT GEOMETRY PARAMETERS OF OUR CBKR STRUCTURES

\begin{tabular}{cccccc}
\hline Geometry & $\begin{array}{c}L(D) \\
\mu \mathrm{m}\end{array}$ & $\begin{array}{c}\delta \\
\mu \mathrm{m}\end{array}$ & $\begin{array}{c}\boldsymbol{\delta}_{\mathbf{U}} \\
\mu \mathbf{m}\end{array}$ & $\begin{array}{c}\boldsymbol{\delta}_{\mathbf{L}} \\
\mu \mathbf{m}\end{array}$ & $\begin{array}{c}V_{\text {TAP }} \\
\mu \mathrm{m}\end{array}$ \\
\hline Square & 1 & $0.2-5$ & $\mathrm{n} / \mathrm{a}$ & $\mathrm{n} / \mathrm{a}$ & $\mathrm{n} / \mathrm{a}$ \\
Square & 2 & $0.2-5$ & $\mathrm{n} / \mathrm{a}$ & $\mathrm{n} / \mathrm{a}$ & $\mathrm{n} / \mathrm{a}$ \\
Square & 4.4 & $0.2-5$ & $\mathrm{n} / \mathrm{a}$ & $\mathrm{n} / \mathrm{a}$ & $\mathrm{n} / \mathrm{a}$ \\
Square & 8.9 & $0.2-5$ & $\mathrm{n} / \mathrm{a}$ & $\mathrm{n} / \mathrm{a}$ & $\mathrm{n} / \mathrm{a}$ \\
Square & 17.7 & $0.2-5$ & $\mathrm{n} / \mathrm{a}$ & $\mathrm{n} / \mathrm{a}$ & $\mathrm{n} / \mathrm{a}$ \\
Square & 8.9 & $\mathrm{n} / \mathrm{a}$ & $0.2-5$ & $0.2-5$ & $\mathrm{n} / \mathrm{a}$ \\
Round & 5 & $0.5-10$ & $\mathrm{n} / \mathrm{a}$ & $\mathrm{n} / \mathrm{a}$ & $1-2$ \\
Round & 10 & $0.5-10$ & $\mathrm{n} / \mathrm{a}$ & $\mathrm{n} / \mathrm{a}$ & $1-2$ \\
Round & 20 & $0.5-10$ & $\mathrm{n} / \mathrm{a}$ & $\mathrm{n} / \mathrm{a}$ & $1-2$ \\
Round & 10 & $\mathrm{n} / \mathrm{a}$ & $1-5$ & $1-5$ & 2 \\
Round & 20 & $\mathrm{n} / \mathrm{a}$ & $1-5$ & $1-5$ & 2 \\
\hline
\end{tabular}

Finally, the structures received 20 -min annealing at $400{ }^{\circ} \mathrm{C}$ in a $\mathrm{N}_{2} / \mathrm{H}_{2}(10 \%)$ mixture. 


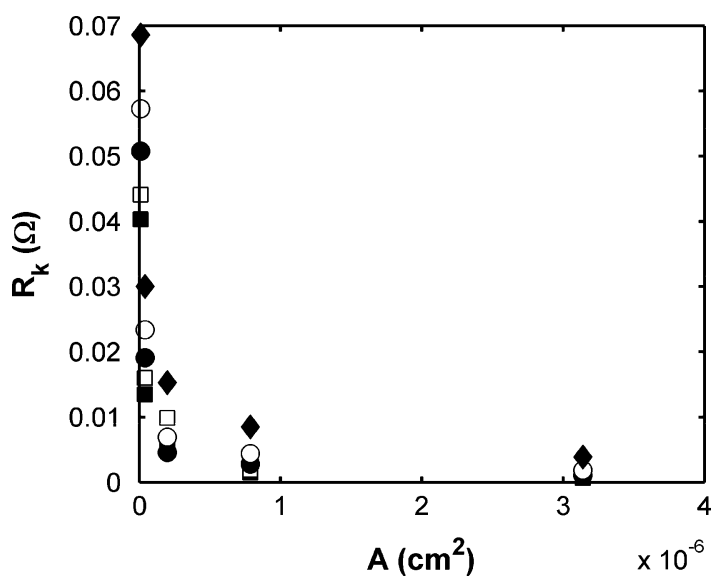

Fig. 3. Measured Kelvin resistance versus contact size for given symmetric overlap area sizes for square contacts: $\delta=0.2 \mu \mathrm{m}(\boldsymbol{\square}), \delta=0.5 \mu \mathrm{m}(\square), \delta=$ $1 \mu \mathrm{m}(\bigcirc), \delta=2 \mu \mathrm{m}(\bigcirc), \delta=5 \mu \mathrm{m}(\diamond)$.

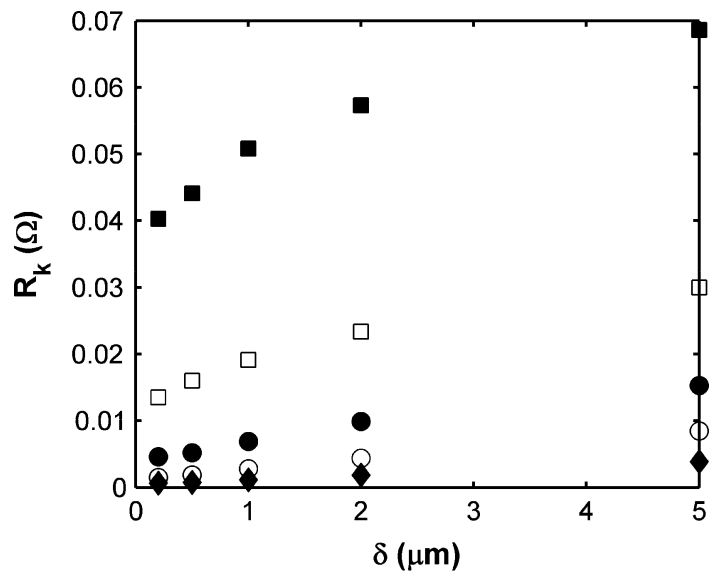

Fig. 4. Measured Kelvin resistance versus symmetric overlap size for given square contact sizes: $L=1 \mu \mathrm{m}(\square), L=2 \mu \mathrm{m}(\square), L=4.4 \mu \mathrm{m}$ ( $)$, $L=8.9 \mu \mathrm{m}(\bigcirc), L=17.7 \mu \mathrm{m}(\bullet)$.

\section{RESULTS AND DISCUSSION}

\section{A. Measured Kelvin Resistance $R_{\mathrm{k}}$ for Square Contacts With Symmetric $\delta$ for Lower and Upper Metals}

The $R_{\mathrm{k}}$ data as a function of contact size $A=L^{2}$ and the overlap size $\delta$ are given in Fig. 3 and Fig. 4, respectively. It can clearly be seen that $R_{\mathrm{k}}$ increases with increasing $\delta$ and decreases with increasing contact size. This is in agreement with the theory (4), demonstrating, that for $\delta>0$, the lateral current flow gives rise to an additional voltage drop that is included in $V_{34}$, leading to a higher $R_{\mathrm{k}}$ value.

\section{B. Measured Kelvin Resistance $R_{k}$ for Square Contacts With Nonsymmetric Overlap Areas for Lower and Upper Metals}

For the nonsymmetric overlaps for upper and lower metals, the $R_{\mathrm{k}}$ values were dependent on the direction of the forced current $I$, as expected. This is in contrast to the other structures with symmetric $\delta$ for lower and upper metals, where $R_{\mathrm{k}}$ was not direction dependent (Fig. 5). It is noteworthy that (4) is derived for extracting specific metal-to-silicon contact resistance, where only $R_{\mathrm{sh}}$ and $\delta$ of the diffusion layer are considered, since $R_{\text {sh }}$ of the metal is much lower than that of even highly doped

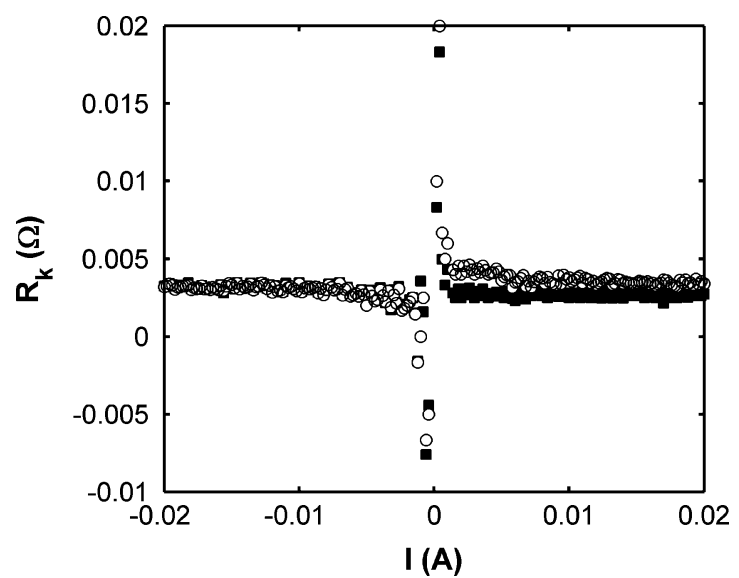

Fig. 5. Measured Kelvin resistance versus forced current for symmetric ( (i.e., $\delta_{\mathrm{L}}=\delta_{\mathrm{U}}$ ) and nonsymmetric (i.e., $\boldsymbol{\delta}_{\mathrm{L}} \neq \delta_{\mathrm{U}}$ ) (O) overlaps of square contacts.

silicon. While measuring contact resistance between two materials with similar $R_{\mathrm{sh}}, \delta$ and $R_{\mathrm{sh}}$ of both layers must be taken into account. Therefore, the $R_{\mathrm{k}}$ dependences on $\delta_{L}$ and $\delta_{U}$ were studied separately [Fig. 6(a) and (b)]. It was demonstrated that $R_{\mathrm{k}}$ values increased with increasing $\delta_{L}$ or $\delta_{U}$.

\section{Measured Kelvin Resistance $R_{\mathrm{k}}$ for Round Contacts}

For round structures, the $R_{\mathrm{k}}$ data for different metal tap widths $\left(V_{\text {tap }}\right)$ as a function of contact size $(A)$ and overlap size $(\delta)$ are given in Figs. 7 and 8, respectively.

Similar to the square contacts, the $R_{\mathrm{k}}$ increased with increasing $\delta$ and decreased with increasing $A$, in agreement with the theory (4). The $R_{\mathrm{k}}$ was not dependent on $V_{\mathrm{tap}}$ (Figs. 7 and 8 ), proving validity of the measurements and supporting a correct definition of overlap size for round contacts. The $R_{\mathrm{k}}$ behavior for the nonsymmetric overlaps was studied by varying $\delta_{L}$ and $\delta_{U}$ separately and revealed the same behavior as for the square contacts.

\section{Extraction of $\rho_{\mathrm{c}}$ Using the Analytical Model of Schreyer and Saraswat}

The specific contact resistance was extracted using both the 1-D and 2-D approximations for a variety of different contact and overlap sizes. The $\rho_{c}$ values for square contacts with symmetric overlaps can be found in Figs. 9 and 10. The $\rho_{\mathrm{c}}$ values obtained using the 1-D approach (Fig. 9) were strongly dependent on the contact and overlap size. This supported the significance of applying the 2-D Model instead of the simple 1-D approximation, discussed earlier. The $\rho_{\mathrm{c}}$ values, extracted using the 2-D Model for the smallest contact sizes, were hardly dependent on the overlap dimensions and revealed similar values for different contact sizes. As the contact size increased, the disagreement with the model appeared, showing difference between the geometrical factor, calculated from (4) and the actual geometrical factor, which led to a clear dependence on $\delta$ (Fig. 10). For structures with nonsymmetrical overlaps, the $\rho_{\mathrm{c}}$ values were extracted by varying $\delta_{L}$ and $\delta_{U}$ separately, using $R_{\text {sh }}$ (4) of the corresponding metal layers. An example for $\delta_{\mathrm{L}}$ can be found in Fig. 11, where the current direction was from the lower to upper metal. If the current direction is changed, the $\rho_{\mathrm{c}}$ extracted 
(a)

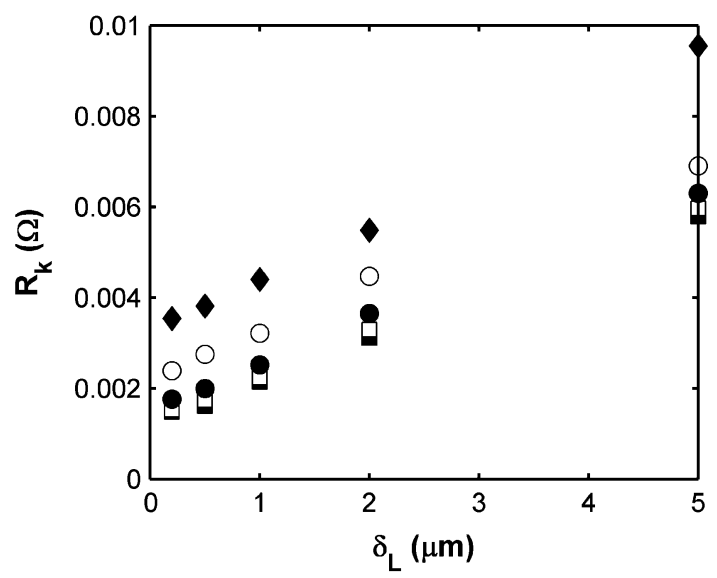

(b)

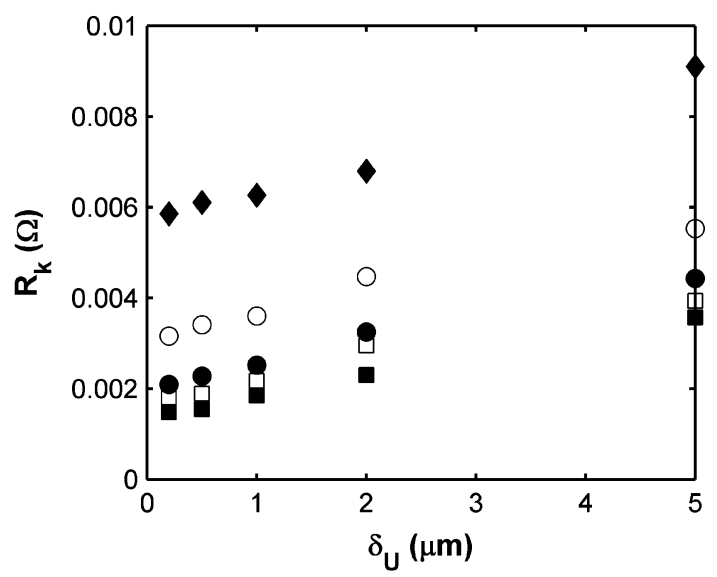

Fig. 6. Dependence of measured Kelvin resistance on overlap size $\delta_{L}$ (a) varying $\delta_{U}$ of $0.2 \mu \mathrm{m}(\mathbf{\square}), 0.5 \mu \mathrm{m}(\square), 1 \mu \mathrm{m}(\mathbf{O}), 2 \mu \mathrm{m}(\bigcirc), 5 \mu \mathrm{m}(\bullet)$ and on overlap size $\delta_{U}$; (b) varying $\delta_{L}$ of $0.2 \mu \mathrm{m}(\mathbf{\square}), 0.5 \mu \mathrm{m}(\square), 1 \mu \mathrm{m}$ $(\bigcirc), 2 \mu \mathrm{m}(\bigcirc), 5 \mu \mathrm{m}(\diamond)$ for given square contact size $(L=8.9 \mu \mathrm{m})$.

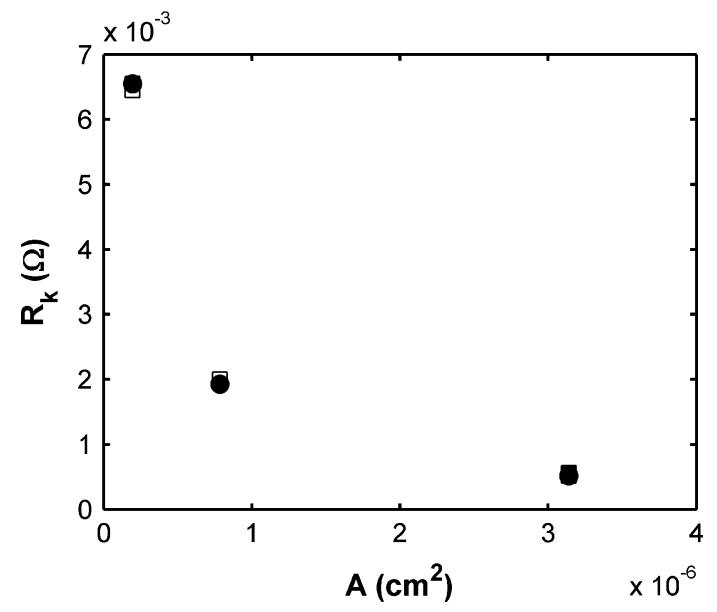

Fig. 7. Measured Kelvin resistance versus contact size for given symmetric overlap area sizes for round contacts and various $V_{\text {tap }}=2 \mu \mathrm{m}(\boldsymbol{\square}), V_{\text {tap }}=$ $1.5 \mu \mathrm{m}(\square), V_{\text {tap }}=1 \mu \mathrm{m}(\mathbf{O})$.

using $\delta_{U}$ (instead of $\delta_{L}$ ) revealed the same values. In summary, it was found that $\rho_{\mathrm{C}}$ is determined by $\delta_{L}$, if current enters from

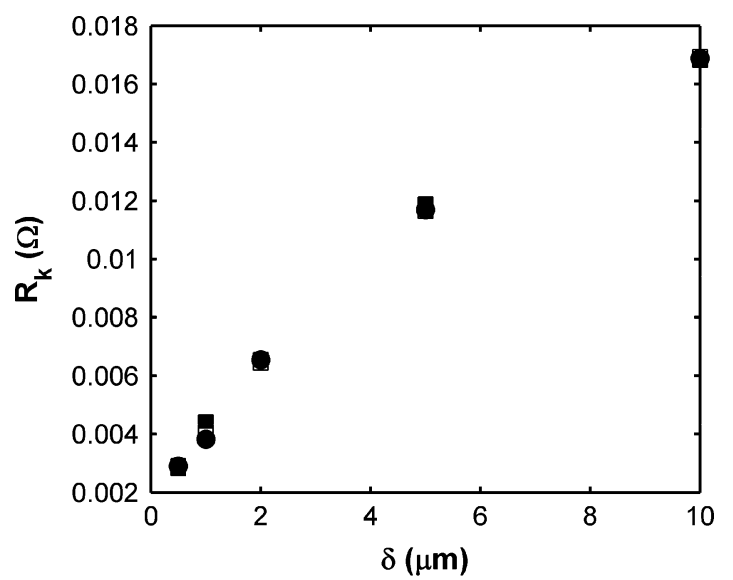

Fig. 8. Measured Kelvin resistance versus symmetric overlap size for given round contact sizes and various $V_{\text {tap }}=2 \mu \mathrm{m}(\boldsymbol{\square}), V_{\text {tap }}=1.5 \mu \mathrm{m}$ $(\square), V_{\text {tap }}=1 \mu \mathrm{m}(\mathbf{O})$.

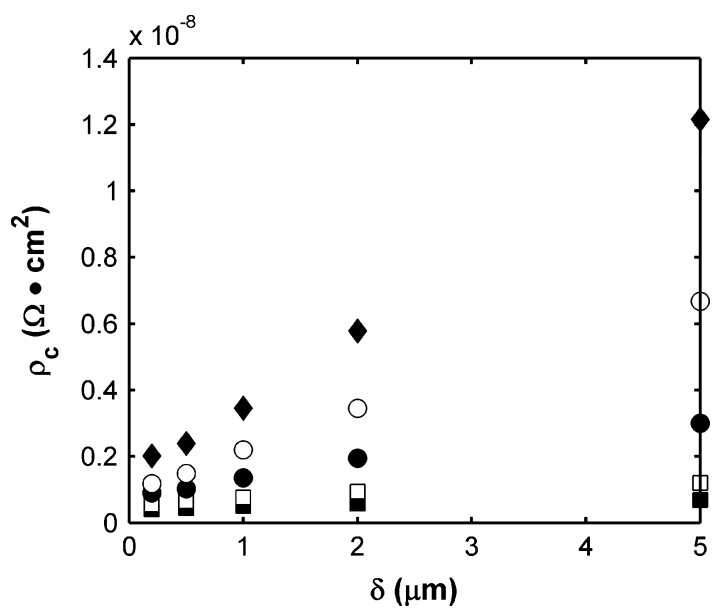

Fig. 9. Specific contact resistance obtained using 1-D approach versus overlap size for given square contact sizes: $L=1 \mu \mathrm{m}(\mathbf{\square}), L=2 \mu \mathrm{m}(\square), L=$ $4.4 \mu \mathrm{m}(\bullet), L=8.9 \mu \mathrm{m}(\bigcirc), L=17.7 \mu \mathrm{m}(\bullet)$.

the lower metal and by $\delta_{U}$ if the current enters from the upper metal.

For a given contact size, the $\rho_{\mathrm{c}}$ values obtained using the 1-D approach were also strongly dependent on the overlap size, in contrast to the values, extracted using the 2-D Model (Fig. 11). The latter was also observed for the round contacts with various $V_{\text {taps }}$ (Fig. 12).

\section{E. Our Approach to Account for the Actual Current Flow Regions}

A more accurate approach to extract the $\rho_{\mathrm{c}}$ value is the extrapolation of the measured dependence $R_{\mathrm{k}}$ versus $\delta$ to $\delta=0$, and the calculation of $\rho_{\mathrm{c}}$ from (2) using the $R_{\mathrm{k}}$ value at $\delta=0$ as the $R_{\mathrm{c}}$. In this manner, the model simplifications, assumed while deriving (4), can be ignored. However, for larger contacts, the results were still dependent on the contact size (Fig. 13). An explanation of this observation is that the current, which contributes to $V_{34}$, can flow across a smaller area compared to the actual contact area $A$. As the contact size becomes larger, this effect enhances, causing significant differences while extracting $\rho_{\mathrm{c}}$. To account for this effect, the potential distributions along 


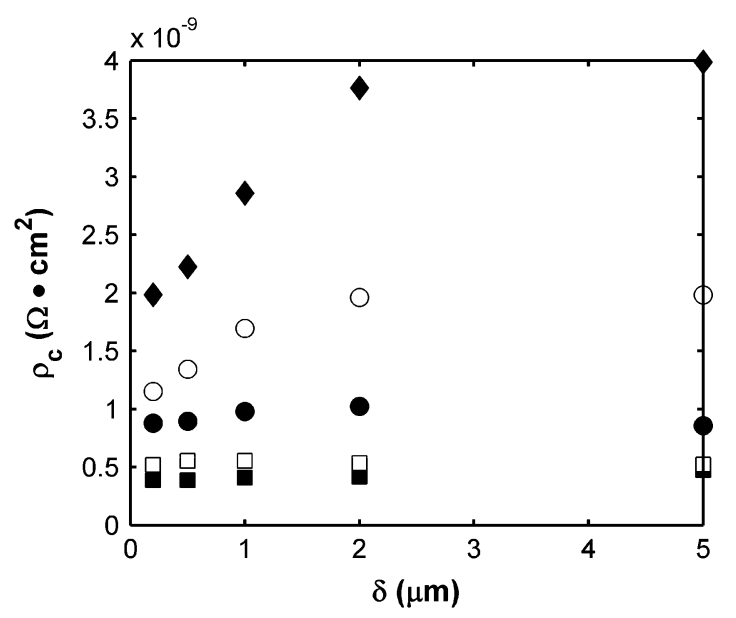

Fig. 10. Specific contact resistance obtained using 2-D approach versus overlap size for given square contact sizes: $L=1 \mu \mathrm{m}(\mathbf{\square}), L=2 \mu \mathrm{m}(\square), L=$ $4.4 \mu \mathrm{m}(\bigcirc), L=8.9 \mu \mathrm{m}(\bigcirc), L=17.7 \mu \mathrm{m}(\bullet)$.

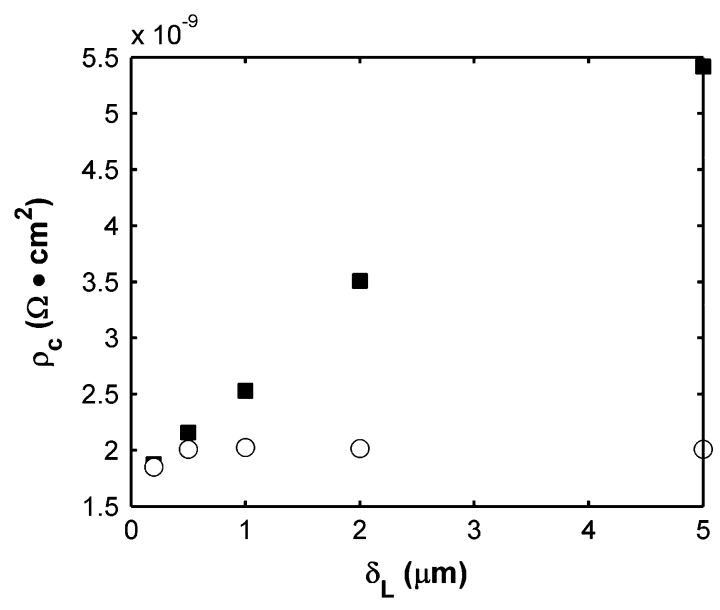

Fig. 11. Specific contact resistance obtained using 1-D $(\square)$ and 2-D $(O)$ approach versus $\delta_{L}$ for given $\delta_{U}=2 \mu \mathrm{m}$ and square contact size $(L=8.9 \mu \mathrm{m})$.

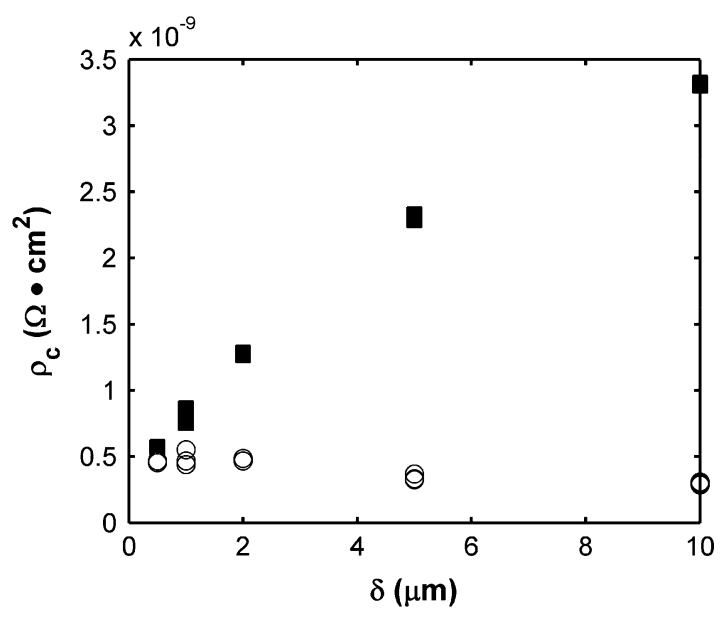

Fig. 12. Specific contact resistance obtained using 1-D ( $\square)$ and 2-D $(O)$ approach versus overlap size for given round contact size.

2 horizontal resistive layers, vertically separated by a resistive "contact," must be considered [10]. For the given test structures,

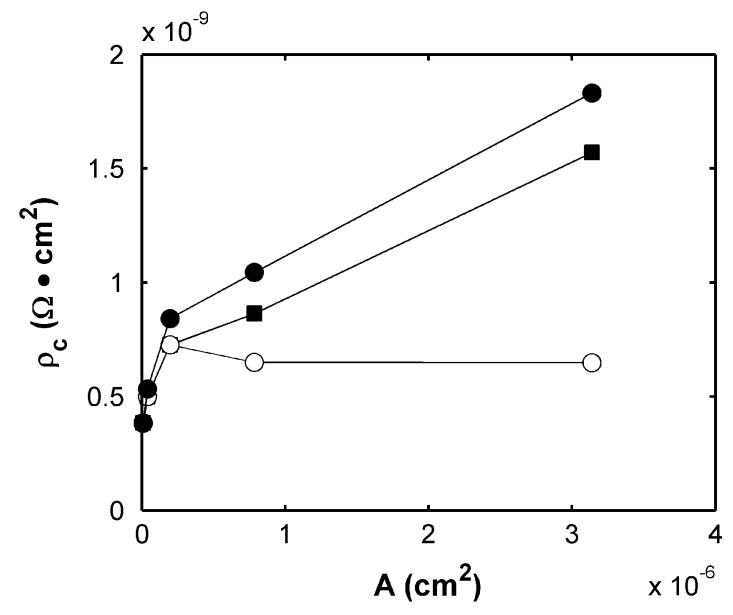

Fig. 13. Specific contact resistance versus contact size obtained using Schreyer and Saraswat model and then extrapolated to $\delta=0(\mathcal{O})$, the extrapolation of $R_{\mathrm{k}}$ to $\delta=0(\square)$ and the current-flow area correction $(\bigcirc)$.

such distributions can be described by the following pair of coupled differential equations:

$$
\begin{aligned}
\frac{\partial^{2} V_{\mathrm{L}}(x)}{\partial x^{2}} & =\frac{R_{\mathrm{shL}}}{\rho_{\mathrm{CG}}}\left(V_{\mathrm{L}}(x)-V_{\mathrm{U}}(x)\right) \\
\frac{\partial^{2} V_{\mathrm{U}}(x)}{\partial x^{2}} & =\frac{R_{\mathrm{shU}}}{\rho_{\mathrm{CG}}}\left(V_{\mathrm{U}}(x)-V_{\mathrm{L}}(x)\right)
\end{aligned}
$$

where $V_{\mathrm{U}}(\mathrm{x})$ and $V_{\mathrm{L}}(\mathrm{x})$ are the potential distributions in the upper and lower metal layers, respectively, $x$ is the coordinate along the contact length $L, R_{\text {shU }}$ and $R_{\text {shL }}$ are the sheet resistances of the upper and lower metals, respectively, and $\rho_{\mathrm{CG}}$ corresponds to the specific resistance caused by the properties and geometry of the contact. Applying the boundary conditions, appropriate for the particular geometry, and using $\rho_{\mathrm{c}}$ as a fitting parameter to obtain the corresponding $R_{\mathrm{k}}$, the $V_{34}(x)=$ $V_{\mathrm{U}}(x)-V_{\mathrm{L}}(x)$ (i.e., voltage difference distribution along the contact) can be calculated. It is important to note that the $V_{34}(x)$ dependence will indicate the actual current flow areas because the current can only flow from the lower metal layer into the upper metal layer if $V_{34}(x) \neq 0$. It was shown that for the small contacts, the current flow area was identical to that of the contact, while for the larger contacts this area was much smaller than the designed contact area (Fig. 14(a)). The demonstrated approach allowed to estimate the actual current-flow area size $A_{\text {cor }}\left(A_{\text {cor }}<A\right)$ and therefore resulted in a corrected $\rho_{\mathrm{c}}$. A comparison of the extraction methods is presented in Fig. 13. Our approach results in similar $\rho_{\mathrm{c}}$ values for various contact sizes. This obviously points to the importance of knowing the actual current flow distribution. The extracted $\rho_{\mathrm{c}}$ values were $(6.31 \pm 0.66) \cdot 10^{-10} \Omega \cdot \mathrm{cm}^{2}$.

The sheet resistances of both the lower and upper metal layers were measured using VDP structures, fabricated on the same wafers. The obtained values of 0.054 and $0.027 \Omega / \square$ for the $0.675-\mu \mathrm{m}$-thick and 1.4- $\mu \mathrm{m}$-thick metals, respectively, were in agreement with the corresponding thicknesses. Due to the fact that the two metals had different thicknesses, the potential distribution along the contact was nonsymmetrical [Fig. 14(a)]. For the wafers, processed with the similar upper and lower metal 
(a)

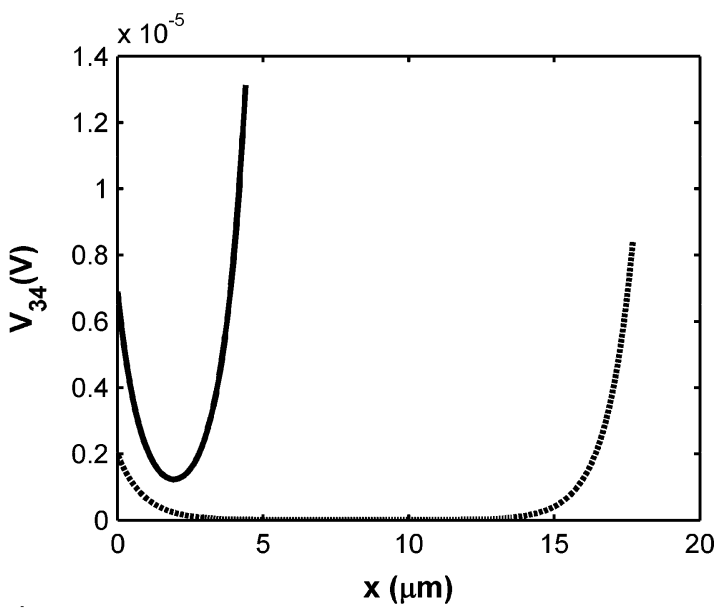

(b)

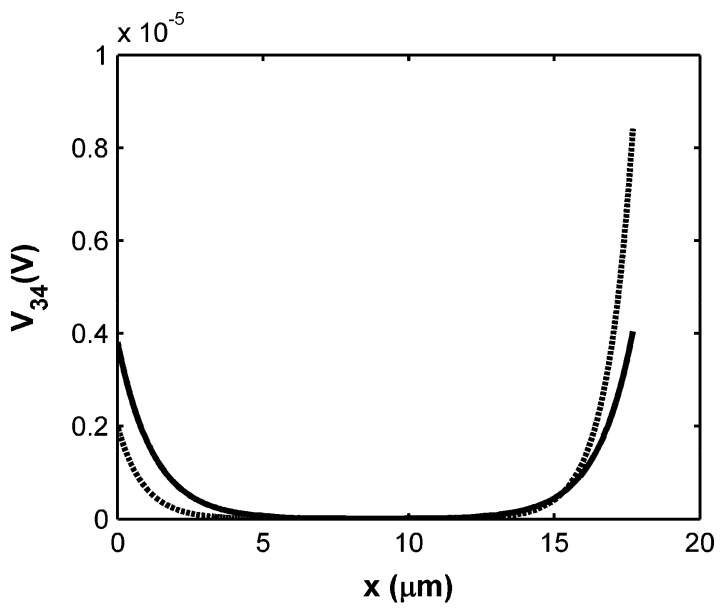

Fig. 14. Potential difference distribution $V_{34}(x)=V_{\mathrm{U}}(x)-V_{\mathrm{L}}(x)$ along the contact coordinate $x$ (a) for the contact length of $4.43 \mu \mathrm{m}$ (solid line) and $17.72 \mu \mathrm{m}$ (dotted line) and differently-thick aluminum as a metal; (b) for aluminum with the same thickness (i.e., $R_{\text {sh }}$ (Upper) $=$ $R_{\mathrm{sh}}$ (Lower) $=0.043 \Omega / \square$, (solid line)) and different thicknesses (i.e., $R_{\text {sh }}($ Upper $)=0.054 \Omega / \square$ and $R_{\text {sh }}$ (Lower) $=0.027 \Omega / \square$, (dotted line) .

thicknesses, the potential distribution became symmetrical. The comparison of both distributions for a certain contact length is presented in Fig. 14(b).

\section{F. Contact Interface Characterization and the Minimum Value of $\rho_{c}$ to be Accurately Extracted}

The measured $R_{\mathrm{k}}$ values for wafers, processed with and without RF pre-clean procedure prior to the deposition of the upper metal were compared (Fig. 15). It was found that, for all contact sizes for the wafers without the RF pre-clean, higher $R_{\mathrm{k}}$ values were measured, indicating presence of the contact interface.

The estimated Kelvin resistance, calculated from the two-metal stack of the known geometry and the sheet resistances, matched with the measured $R_{\mathrm{k}}$ values for the wafers, processed with the RF pre-clean (i.e., having "ideal" contacts). Thus, it provided the minimum value of $\rho_{\mathrm{c}}$ to be accurately

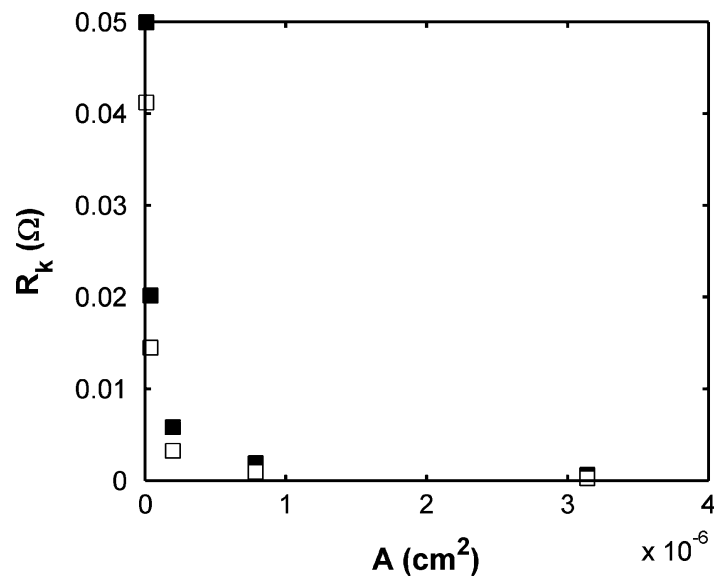

Fig. 15. Measured $R_{\mathrm{k}}$ values for the wafers with ( $\square$ ) and without ( $\square$ ) RF pre-clean procedure prior to the upper metal deposition.

extracted from the given CBKR structures, and the method for contact interface characterization. In this work, the aluminum-aluminum contacts have been analyzed. For the present technology nodes using copper metallization, the properties of, e.g., the $\mathrm{Cu}-\mathrm{Ta}(\mathrm{N})-\mathrm{Cu}$, contact structures can be described accordingly.

\section{CONCLUSION}

A design and fabrication of various metal-to-metal CBKR structures has been realized. The structures included a large variety of contact geometries, i.e., various shapes and sizes for contact holes and overlap regions. The obtained Kelvin resistance, $R_{\mathrm{k}}$, was in agreement with the analytical model proposed by Schreyer and Saraswat. This demonstrated the necessity to account for 2-D current flow effects around the contact area while measuring low contact resistance values. However, as the calculated $\rho_{\mathrm{c}}$ values were still dependent on the contact size, we developed a new correction method to account for the actual current-flow areas through the contact. The approach allowed to obtain a potential difference distribution along the contact length and led to a physically-correct extraction of the $\rho_{\mathrm{c}}$. The measured $R_{\mathrm{k}}$ values for the wafers processed with RF pre-clean procedure corresponded to the two-metal stack resistance, calculated from the given dimensions of the contact size and sheet resistances of the metals used. The measured $R_{\mathrm{k}}$ values for the wafers processed without RF pre-clean procedure were higher, indicating presence of the contact interface. This can be used to improve contact process characterization in contact manufacturing. As a result, the minimum value to be accurately extracted from the CBKR structures was determined.

\section{ACKNOWLEDGMENT}

The authors would like to thank the DIMES Clean room staff of Delft University of Technology for processing the wafers.

\section{REFERENCES}

[1] , S. Wolf and R. N. Tauber, Eds., Silicon Processing for the VLSI Era. Sunset Beach, CA: Lattice Press, 1990, vol. 2. 
[2] D. K. Schroder, Semiconductor Material and Device Characterization, 3rd ed. New York: Wiley-Interscience/IEEE, 2006.

[3] W. M. Loh, S. E. Swirhun, E. Crabbe, K. Saraswat, and R. M. Swanson, "An accurate method to extract specific contact resistivity using crossbridge Kelvin resistors," IEEE Electron Device Lett., vol. EDL-6, no. 9, pp. 441-443, Sep. 1985.

[4] T. A. Schreyer and K. C. Saraswat, "A two-dimensional analytical model of the cross-bridge Kelvin resistor," IEEE Electron Device Lett., vol. EDL-7, no. 12, pp. 661-663, Dec. 1986.

[5] J. Santander, M. Lozano, A. Collado, M. Ullan, and E. Cabruja, "Accurate contact resistivity extraction on Kelvin structures with upper and lower resistive layers," IEEE Trans. Electron Devices, vol. 47, no. 7, pp. 1431-1439, Jul. 2000.

[6] A. S. Holland, G. K. Reeves, and P. W. Leech, "Universal error corrections for finite semiconductor resistivity in Cross-Kelvin resistor test structures," IEEE Trans. Electron Devices, vol. 51, no. 6, pp. 914-919, Jun. 2004.

[7] R. L. Gillenwater, M. J. Hafich, and G. Y. Robinson, "Extraction of the minimum specific contact resistivity using Kelvin resistors," IEEE Electron Device Lett., vol. EDL-7, no. 12, pp. 674-676, Dec. 1986.

[8] M. Finetti, A. Scorzoni, and G. Soncini, "Lateral current crowding effects on contact resistance measurements in 4 terminal resistor test patterns," IEEE Electron Device Lett., vol. EDL-5, no. 12, pp. 524-526, Dec. 1984.

[9] A. Scorzoni, M. Finetti, K. Grahn, I. Suni, and P. Cappelletti, "Current crowding and misalignment effects as sources of error in contact resistivity measurements. 1. Computer-simulation of conventional CER and CKR structures," IEEE Trans. Electron Devices, vol. ED-34, no. 3, pp. 525-531, Mar. 1987.

[10] D. B. Scott, W. R. Hunter, and H. Schichijo, "A transmission-line model for silicided diffusions-Impact on the performance of VLSI circuits," IEEE Trans. Electron Devices, vol. ED-29, no. 4, pp. 651-661, Apr. 1982.

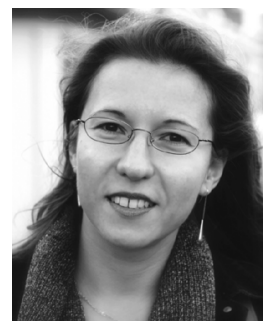

Natalie Stavitski (S'07) received the B.Sc. degree in chemistry from the Hebrew University of Jerusalem, Israel, and the M.Sc. degree in materials chemistry from the Weizmann Institute of Science, Rehovot, Israel, in 2000 and 2003, respectively. She is currently pursuing the Ph.D. degree from the Chair of Semiconductor Components, University of Twente, Enschede, The Netherlands.

From 2002 to 2005, she was a member of engineering staff with the Intel Israel Corporation, where she worked in the area of photolithography processes. Her current research is focused on the conduction mechanism in metal-semiconductor junctions and the development of dedicated test structures for the characterization.

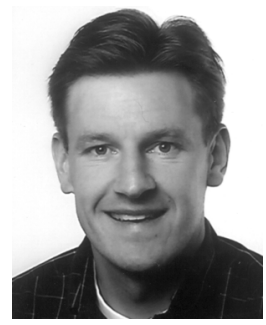

Johan H. Klootwijk (S'95-M'98-SM'08) was born in Hengelo, The Netherlands, on June 2, 1969. He received the M.Sc. and Ph.D. degrees in electrical engineering from the University of Twente, Enschede, The Netherlands, in 1993 and 1997, respectively.

In October 1997, he joined the Philips Research Laboratories, Eindhoven, The Netherlands, where he was involved in the development and characterization of $\mathrm{Si}$ and $\mathrm{SiGe}$ bipolar transistors, SOI/SOA technologies, reliability of thin dielectrics and development of InP-based HBTs wideband RF applications. Since 2004, he has been working on the development, characterization and integration of high-density 3-D devices, in particular capacitors. Currently, he is responsible for technology and test structrure development, in particular for integrated 3-D all-solid-state batteries and bio-nanosensors.

Dr. Klootwijk received the Best Paper Award for his contribution on the ESSDERC Conference in 2001. He served as the Tutorial Chairman of the ICMTS 2002, and 2008 .

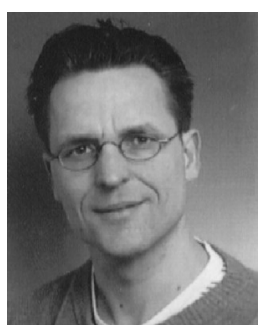

Henk W. van Zeijl studied at the Poly Technical Institute Rijswijk where he received the B.S. degree in physics in 1981. In 2005, he received the Ph.D. degree from Delft University of Technology, Delft, The Netherlands, based on research on bipolar transistors with self aligned emitter-base metallization and backwafer-aligned collector contacts.

In 1981, he joined the Interuniversity Reactor Institute, Delft, where he worked in the field of neutron diffraction and instrumental neutron activation analysis. In 1986, he joined the Delft Institute of Microelectronics and Submicron Technology (DIMES). From 1989 to 1998, he was responsible for the mask fabrication and lithography in the DIMES IC process research sector. During that period he assisted in different research programs. In 1998, he developed a lithography course "Applied I-Line Lithography," and two years later the course "Integrated Circuit Fabrication Technology." These courses, both full week training for engineers from relevant fields in the industry, are held for more than forty times and are also transferred to the Tshinghua University, Beijing, China. Besides these educational activities, he cooperated in different research projects related to lithography and MEMs. He is now a senior researcher at DIMES, and is involved in different research projects related to MEMs and 3-D integration. He was recently appointed as a lecturer and is a (co-)author of more than 40 technical papers.

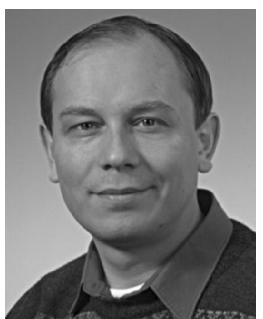

Alexey Y. Kovalgin received the M.Sc. degree in physics from St. Petersburg State University, St. Petersburg, Russia, in 1988, and the Ph.D. degree in electronic materials technology from St. Petersburg State Polytechnical University, St. Petersburg, in 1995.

In 1997, he joined the University of Twente, Enschede, The Netherlands, as a Postdoctoral Researcher. Since 2001, he has been an Assistant Professor at the Chair of Semiconductor Components, University of Twente, where he is involved in thin-film deposition technologies (CVD, ALD, plasma processing, modelling, thin-film characterization), design, and realization and characterization of novel silicon devices. He contributed to over 90 reviewed international journal and conference papers.

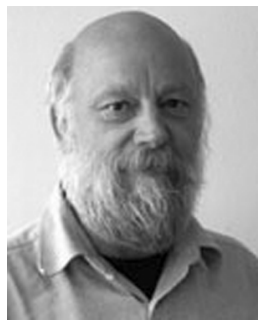

Rob A. M. Wolters received the M.Sc. degree in inorganic chemistry from the University of Twente, Enschede, The Netherlands, in 1974 and the Ph.D. degree based on the work on uraniumcarbonitrides at the Reactor Centrum Nederland, Petten, The Netherlands, in 1978.

He has been with Philips Research (currently at NXP Research), Eindhoven, The Netherlands, where he covered a large number of subjects related to the processing of Si integrated circuits. He has been involved in the introduction of chlorine-based plasma etching processes for gates and interconnects. He has vast knowledge of the application of silicides, barrier materials and metals in the Si technology area. Since 2004, he has been a part-time Professor with the Chair of Semiconductor Components, University of Twente, Enschede, The Netherlands. 